\title{
REAC Non-invasive Neurobiological Stimulation for Mitigating the Impact of Internalizing Disorders in Autism Spectrum Disorder
}

\author{
Arianna Rinaldi ${ }^{1,2} \cdot$ Margherita Maioli ${ }^{1,2} \cdot$ Márcia C. Marins Martins ${ }^{3}$. Pollyanna Celso Felipe de Castro ${ }^{3}$. \\ Natália Almeida Prado de Oliveira Silva ${ }^{3}$ Jaqueline Albieri Vieira de Mattos ${ }^{3}$ Vania Fontani ${ }^{2,4}$. \\ Salvatore Rinaldi ${ }^{2,4}$ (]
}

Accepted: 11 September 2021 / Published online: 21 September 2021

(c) The Author(s) 2021

\begin{abstract}
Objectives Allostatic overload occurs when environmental factors become excessive for the individual capacity of neurobiological response and can trigger internalizing disorders. These disorders have a strong impact on individuals with autism spectrum disorder (ASD) and can exacerbate ASD symptoms and deficits. A non-invasive neurobiological stimulation technology, called the radio electric asymmetric conveyer (REAC), has been shown to be effective in improving internalizing disorders and remodeling the individual allostatic response. On these premises, REAC neurobiological stimulation treatments have been introduced for the treatment of internalizing disorders in ASD. The aim of this current research was to retrospectively verify the effects of REAC treatments on motor, cognitive, and behavioral skills and symptoms, assessed using the Autism Profiling Questionnaire (APQ).

Methods Data were collected through administration of the APQ to the caregivers of 112 individuals with ASD. The caregivers rated every cluster of the questionnaire with a score based on an evaluation scale ranging from 0 to 10 .

Results At the 3-month follow-up, the caregivers perceived and rated a global improvement of the individuals with ASD in all clusters.

Conclusions This research suggests that the mitigating effects of REAC neuromodulation treatments in relation to the severity of internalizing disorders can enhance the quality of life and the autonomy of individuals with ASD, as shown by the shift in the median values in all 21 items of the APQ toward an improvement in symptoms and skills.
\end{abstract}

Keywords Autism $\cdot$ Anxiety $\cdot$ Depression $\cdot$ Neurobiological stimulation $\cdot$ Allostatic overload $\cdot$ Internalizing disorders

Autism spectrum disorder (ASD) is a neurobiological disorder with an incidence that is growing worldwide. Therefore, there is a need to develop therapeutic strategies to improve the quality of life of individuals with ASD. The comorbidity

Salvatore Rinaldi

srinaldi@irf.it

1 Department of Biomedical Sciences, University of Sassari, Sassari, Italy

2 Department of Adaptive Neuro Psycho Physio Pathology and Neuro Psycho Physical Optimization, Rinaldi Fontani Institute, Florence, Italy

3 International Scientific Society of Neuro Psycho Physical Optimization With REAC Technology, Brazilian Branch, São Paulo, Brazil

4 Research Department, Rinaldi Fontani Foundation, Florence, Italy of internalizing disorders, such as depression (DeFilippis, 2018; Gegelashvili, 2019), anxiety (Adams \& Emerson, 2021; Vasa et al., 2020), and stress-related disorders (Oerbeck et al., 2021; Taylor et al., 2021), with ASD is very often present and can affect the deficit severity and the overall quality of life of individuals with ASD. Some non-invasive brain stimulation (NIBS) techniques based on different technologies, such as transcranial magnetic stimulation (Enticott et al., 2019) (TMS) and transcranial direct current stimulation (Amatachaya et al., 2014; Garcia-Gonzalez et al., 2021) (TDCS), have been proven to induce improvements in social, health, and behavioral problems among these individuals.

Another non-invasive technique for neurobiological stimulation, called the radio electric asymmetric conveyer (REAC), has been proven in several studies to be effective in psychological and psychiatric disorders, classified in the DSM-5 as internalizing disorders, including depression 
(Olivieri et al., 2011; Rinaldi et al., 2019), anxiety (Fontani et al., 2011a, 2011b; Pinheiro Barcessat et al., 2020a, 2020b), stress-related disorders (Fontani et al., 2011a, 2011b; Rinaldi et al., 2010), and motor skills (Lorenzini et al., 2016; Olazaran et al., 2014), balance (Fontani et al., 2012), and other psychiatric disorders (Fontani et al., 2011a, 2011b). As these disorders can exacerbate ASD symptoms and deficits, REAC neurobiological stimulation treatments have been used for some years in individuals with ASD to address their social, health, and behavioral problems. The current research is aimed to retrospectively analyze the treatment results collected in a standardized manner using a specific test designed to evaluate the effects of REAC neurobiological stimulation treatments on the degree of expression of some motor, cognitive, and behavioral deficits typical of ASD and conditioned by internalizing disorders.

\section{Method}

\section{Participants}

The total population included in this international research consisted of 112 children with ASD with an average age of $5.96 \pm 3.15$ years, including 26 girls with an average age of $7 \pm 3.41$ years and 86 boys with an average age of $5.65 \pm 3.01$ years. This population consisted of all children who visited the clinical centers of the International Scientific Society of Neuro Psycho Physical Optimization with REAC Technology (SONC) for over 12 months and who had a previous diagnosis made by a child psychiatrist using the Autism Diagnostic Observation Schedule (ADOS) and Autism Diagnostic Interview-Revised (ADI-R). Besides the existing treatment plans, the children's caregivers voluntarily opted to add the REAC neuromodulation treatments.

\section{Procedure}

The REAC neuromodulation treatments were administered at the SONC clinical facilities, and data were collected for over 12 months.

\section{REAC Technology}

The REAC technology for neurobiological stimulation is aimed at optimizing the endogenous bioelectrical activity (EBA) of neural circuits (Baer \& Colello, 2016; Levin et al., 2017). EBA generates signals involved in epigenetic cascades and transcriptional changes in cellular properties, such as proliferation, differentiation, migration, shape change, senescence, apoptosis, neuroinflammation, and neurotransmission (Chernet \& Levin, 2013; Levin, 2014). The EBA mechanism implies that an asymmetrical gradient exists in all the components of an organism, which allows the creation of ionic flows, consequently producing currents and endogenous bioelectric fields (EBFs) (Tuszynski, 2019). Many mechanisms, including exposome pressure, can invalidate the correct and adequate production of ionic flows and therefore EBFs, causing a wide range of health problems. Because EBFs are also involved in neurotransmission mechanisms, their alteration can cause mood and behavioral problems. The optimization of EBA through the REAC technology is made possible because REAC devices emit radio electric fields in the environment. These radio electric fields interact with an individual's body through the device's asymmetric conveyer probe (ACP). This interaction promotes the formation of endogenous gradients that promote the release of charge densities induced by external factors, thus facilitating neuromodulation processes. The data reported by various studies confirm that the REAC technology, used according to specific protocols, can optimize EBA and induce direct cell reprogramming (Maioli et al., 2012, 2013), contrast of senescence (Maioli et al., 2014; Rinaldi, Maioli, et al., 2014), contrast of neuroinflammation (Lorenzini et al., 2016; Panaro et al., 2018), and modulation of neurotransmission even in neurodegenerative diseases (Lorenzini et al., 2016; Rinaldi et al., 2015).

\section{REAC Neuromodulation Treatments}

In this study, one session of neuro postural optimization (NPO) treatment (Olazaran et al., 2014; Rinaldi et al., 2014a, 2014b) and one cycle of neuro psycho physical optimization (NPPO/NPPO-CB) treatments (Pinheiro Barcessat et al., 2020a, 2020b; Rinaldi et al., 2019) were administered to mitigate the internalizing disorders that affected the ASD status and quality of life of the children. REAC NPO neuromodulation is a preprogrammed, one-session treatment. It was designed to induce a greater efficiency of the modulatory capacities of the brain response (Mura et al., 2012; Rinaldi et al., 2014a, 2014b) to improve motor control and treat functional dysmetria (FD) (Mura et al., 2012; Rinaldi et al., 2014a, 2014b) even in neurodegenerative diseases, such as Parkinson's and Alzheimer's disease (Olazaran et al., 2013). Its therapeutic result is long-lasting, remaining stable after many years.

NPPO treatments were designed to optimize the individual response to environmental stressors, which can induce and sustain a wide range of dysfunctional adaptive behavioral patterns that underlie many internalizing disorders (Pinheiro Barcessat et al., 2020a, 2020b; Rinaldi et al., 2019). NPPO can be administered as follows: (1) NPPO is administered with a punctiform ACP on the auricle and (2) NPPO-CB with a planar ACP in the cervicobrachial region (Pinheiro Barcessat, et al., 2020a, 2020b). These two methods of administration, both non-invasive 
and equivalent in effectiveness (Pinheiro Barcessat et al., 2020a, 2020b), allow the selection of the option most tolerated by young individuals according to their degree of cooperation. Both NPPO treatments are usually administered in cycles of 18 sessions. The NPPO treatment session lasts for approximately $30 \mathrm{~s}$, while the NPPO-CB session lasts for approximately $4 \mathrm{~min}$. The minimum time interval between the two sessions is $1 \mathrm{~h}$.

The maximum number of sessions per day is usually four. The number of cycles to be administered depends on the individual's condition. In this current research, the REAC treatment cycle was administered within approximately 3 weeks. The parameters for administering these neuromodulation treatments are set by the manufacturer of the device and cannot be modified by the operators. The models of the equipment used for the administration of the treatments were the BENE 101 and BENE 110 models produced by ASMED SRL (Florence, Italy).

The study time points were scheduled as follows: Autism Profiling Questionnaire (APQ) administration (T0), dysmetria assessment (T1), NPO administration (T2), dysmetria assessment after NPO (T3), NPPO/ NPPO-CB administration (T4), dysmetria assessment at follow-up (approximately 3 months after the end of the treatment cycle) (T5), and APQ assessment at follow-up to the children's parents (T6).

\section{Measures}

The effectiveness of the neurobiological stimulation treatments NPO and NPPO/NPPO-CB on the degree of expression of some motor, cognitive, and behavioral deficits typical of ASD was assessed using the APQ and an evaluation of neurological semiology based on the analysis of FD. The APQ is used at the clinical facilities that adhere to the SONC and is administered to the parents or caregivers of children with ASD. It is not a diagnostic test but only a grid for collecting data on the presence and degree of expression of some motor, cognitive, and behavioral deficits typical of ASD in a standardized manner. The questionnaire includes 21 questions or items divided into three clusters: motor skills, cognitive skills, and behavioral skills and symptoms. Table 1 lists the APQ items and clusters. Herein, the caregivers of the children with ASD rated each item of the questionnaire with a score based on an evaluation scale ranging from 0 to 10 (Table 1). The APQ was administered at follow-up (approximately 3 months after the end of the treatment cycle) (T6). The results of the APQ at T0 and T6 were compared to determine which changes the parents observed in their children after the REAC NPO and NPPO neuromodulation treatments.

FD analysis was also performed. Dysmetria is defined as the inability to perform accurate movements owing to injury or dysfunction of the cerebellum (Manto, 2009). The

Table 1 SONC autistic profiling questionnaire

\begin{tabular}{|c|c|c|c|}
\hline Cluster & & Item & Evaluation scale \\
\hline \multirow[t]{9}{*}{ Cognitive skills } & 1 & Language production & \multirow[t]{9}{*}{ Evaluation scale from 0 (no skill) to 10 (very good skill) } \\
\hline & 2 & Language comprehension & \\
\hline & 3 & Attention concentration & \\
\hline & 4 & Learning memory & \\
\hline & 5 & Executive functions & \\
\hline & 6 & Personal autonomy & \\
\hline & 7 & Sphincteric control & \\
\hline & 8 & Night rest & \\
\hline & 9 & Sensory issues & \\
\hline \multirow[t]{2}{*}{ Motor skills } & 10 & Gross motor skills & \\
\hline & 11 & Fine motor skills & \\
\hline \multirow[t]{5}{*}{ Behavioral skills } & 12 & Non-verbal communication & \\
\hline & 13 & Eye contact & \\
\hline & 14 & Adaptation to changes & \\
\hline & 15 & Ability to follow social rules in an interaction & \\
\hline & 16 & Interest in socialization and social contact & \\
\hline \multirow[t]{5}{*}{ Behavioral symptoms } & 17 & Self-aggressive behaviors & \multirow{5}{*}{$\begin{array}{l}\text { Evaluation scale from } 0 \text { (no symptom) to } 10 \text { (high } \\
\text { severity of the symptom) }\end{array}$} \\
\hline & 18 & Hetero-aggressive behaviors & \\
\hline & 19 & Agitation-hyperactivity & \\
\hline & 20 & Irritability—oppositive behavior & \\
\hline & 21 & Stereotypies & \\
\hline
\end{tabular}


cerebellum is initially associated exclusively with control of movements but has been known to play a fundamental role in regulating the adequacy of emotional, behavioral, cognitive, and relational responses (Timmann \& Daum, 2007). This role can be highlighted through the assessment of dysmetria. When dysmetria is present in healthy individuals (Rinaldi et al., 2014a, 2014b), it is defined as functional (Mura et al., 2012) and is correlated with adaptive phenomena at the base of fluctuating asymmetry. On these premises, FD represents one of the main signs of a neuropsycho-physio-pathological and dysfunctional adaptive state and can be an epigenetic measure of stress (Parsons, 1990). FD can be observed through an evaluation of neurological semiology, aimed at highlighting the asymmetric activation of symmetrical muscle groups, such as the quadriceps muscles (Mura et al., 2012; Rinaldi et al., 2014a, 2014b). Postmortem (Hampson \& Blatt, 2015) and neuroimaging studies (D'Mello et al., 2015; Hashimoto et al., 1995) reported cerebellar anomalies of the anatomical and functional types, respectively, in individuals with ASD (Johnson et al., 2014). Therefore, ASD can be defined as a neurobiological disorder related also to both cerebellar connectivity and dysfunction (Becker \& Stoodley, 2013). This impacts the brain areas that govern the cognitive functions, including language and executive functions, as well as affective regulation (Becker \& Stoodley, 2013; D'Mello \& Stoodley, 2015). The NPO treatment, which can determine a long-lasting disappearance of FD in a single administration (Rinaldi et al., 2014a, 2014b), can serve as an important tool in reducing some ASD deficits. In fact, immediately after the administration of NPO, a stable disappearance of FD and an overall reorganization of postural and motor controls are also observed in neurodegenerative diseases (Fontani et al., 2012).

\section{Data Analysis}

Statistical analysis was performed using SPSS version 22 (IBM SPSS). The data collected on the APQ were analyzed using the Wilcoxon and sign tests, performed for each item. Statistical significance was set at $P$-values of $<0.5$. A confidence level of $95 \%$ was selected, in association with confidence intervals (CIs), which were specifically identified for each item of each of the three clusters of the APQ. Each CI was set as the mean value of $\pm 10 \%$.

\section{Results}

\section{FD Evaluation}

The FD evaluation performed immediately after the administration of NPO treatment (T3) showed the disappearance of FD in $100 \%$ of the individuals with ASD. At the 3-month follow-up (T5), the stable disappearance of FD was verified in $100 \%$ of the individuals with ASD.

\section{APQ Results}

The variations between the data collected at $\mathrm{T} 0$ and $\mathrm{T} 6$ were analyzed separately for each of the three clusters: motor skills, cognitive skills, and behavioral skills and symptoms. The data were represented using box plots, a method for graphically depicting groups of numerical data through quartiles. Quartiles divide a rank-ordered dataset into four equal parts. The values that separate the parts are called the first, second, and third quartiles and are denoted by Q1, Q2, and Q3, respectively. The interquartile range (IQR) indicates the distance between the first quartile and the third quartile $(\mathrm{Q} 1-\mathrm{Q} 3)$ and measures how spread out the data points are from the mean of the data set. It is graphically represented by the height of the box: The higher the IQR, the more spread out the data points; in contrast, the smaller the IQR, the more bunched up the data points are around the mean. Box plots may also have lines extending from the boxes (whiskers), indicating variability outside the upper and lower quartiles. The height of the box plot indicates the values within which $50 \%$ of the measured values are contained, while the length of the whiskers represents the tails of the distribution: The greater their length, the greater the dispersion of the values below the first quartile and/ or above the third quartile. To isolate potential outliers, we used Tukey's fences method, which is a nonparametric method developed to detect observations outside the normal range using IQRs. The ranges between $\mathrm{Q} 2-k(\mathrm{Q} 3-\mathrm{Q} 1)$ and $\mathrm{Q} 2+k(\mathrm{Q} 3-\mathrm{Q} 1)$ are referred to as fences. Data points that are below the lower fence or above the higher fence are identified as outliers. The value $k$ determines the width of the fences. The larger the value of $k$, the lower the number of outliers that will be detected. In our analysis, we set the value of $k$ as 1.5. The box plot was used because it was the best visual tool to determine how much the values were dispersed from the median value in the pre- and post-treatment data. Moreover, it allowed the identification of the outliers rapidly and accurately, in comparison with other numerical indices, such as the mean and standard deviation. In fact, if there were anomalous values, these would be represented in the boxplot as isolated asterisks positioned above and/ or below the distribution whiskers. This feature allowed us to highlight very interesting observations, considering how profoundly the profiles of individuals with ASD can differ.

In the behavioral skill and symptom clusters, two distinct box plots were prepared for better visualization of the T0/T6 comparison. In fact, since 5 of the 10 items of this cluster aim to evaluate a skill, the possible improvement of individuals with ASD post-treatment can be detectable if and to the extent in which an increase in values is observed. 
In contrast, since the other five items of the cluster aim to detect the presence of a symptom and evaluate its degree of severity, the possible improvement is detectable if and to the extent to which a reduction in values is observed. A statistical significance of $P<0.5$ was found for the five items aimed at detecting the presence of behavioral symptoms and $P<0.05$ for all the other 16 items.

\section{Cluster Motor Skills}

\section{Gross Motor Skills}

At T0, the Q1 value was 4, and the median value was 6; the Q3 value was 8, and the IQR was 4. At T6, the Q1 value was 5, and the median value was 7; the Q3 value was 8, and the IQR was 3 (Fig. 1). Statistical significance was set at $P$-values of $<0.05$.

\section{Fine Motor Skills}

At T0, the Q1 value was 2, and the median value was 4,5; the Q3 value was 7, and the IQR was 5. At T6, the Q1 value was 5, and the median value was 6; the Q3 value was 8, and the IQR was 3 (Fig. 1). Statistical significance was set at $P$-values of $<0.05$.

Fig. 1 Gross and fine motor skills show an improvement with statistical significance $p<0.05$

\section{Cluster Cognitive Skills}

\section{Language Production Skills}

At T0, the Q1 value was 1.75, and the median value was 4; the Q3 value was 5, and the IQR was 3.25. At T6, the Q1 value was 3, and the median value was 6; the Q3 value was 8 , and the IQR was 5 (Fig. 2). Statistical significance was set at $P$-values of $<0.05$.

\section{Language Comprehension Skills}

At T0, the Q1 value was 3, and the median value was 5; the Q3 value was 7, and the IQR was 4. At T6, the Q1 value was 4, and the median value was 6; the Q3 value was 8, and the IQR was 4 (Fig. 2). Statistical significance was set at $P$-values of $<0.05$.

\section{Attention and Concentration}

At T0, the Q1 value was 3, and the median value was 5; the Q3 value was 5, and the IQR was 2. At T6, the Q1 value was 4 , and the median value was 6; the Q3 value was 7.25, and the IQR was 3.25 (Fig. 2). Statistical significance was set at $P$-values of $<0.05$.

\section{Learning and Memory}

At T0, the Q1 value was 3, and the median value was 5; the Q3 value was 7, and the IQR was 4. At T6, the Q1 value was 5, and the median value was 6; the Q3 value was 8, and

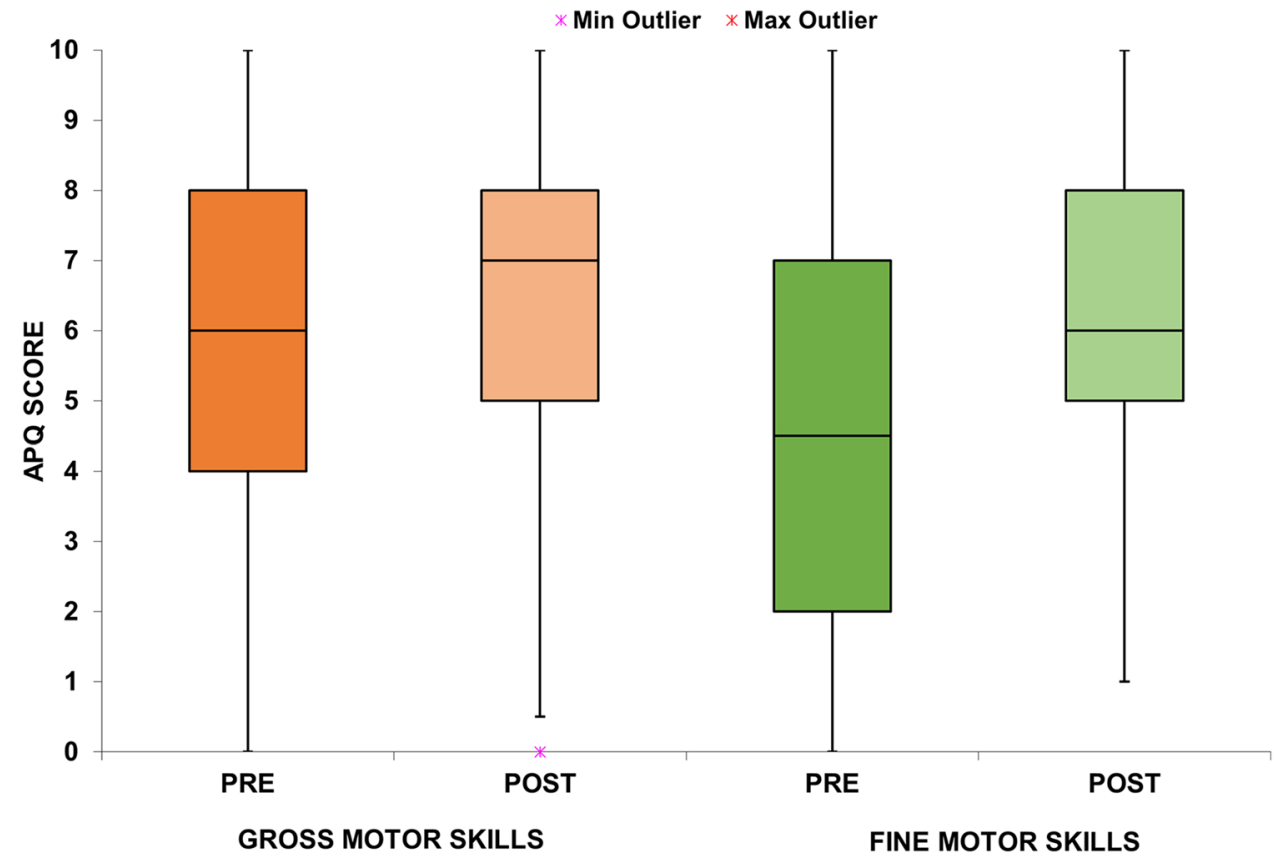


Fig. 2 Cognitive skills show an improvement with statistical significance $p<0.05$

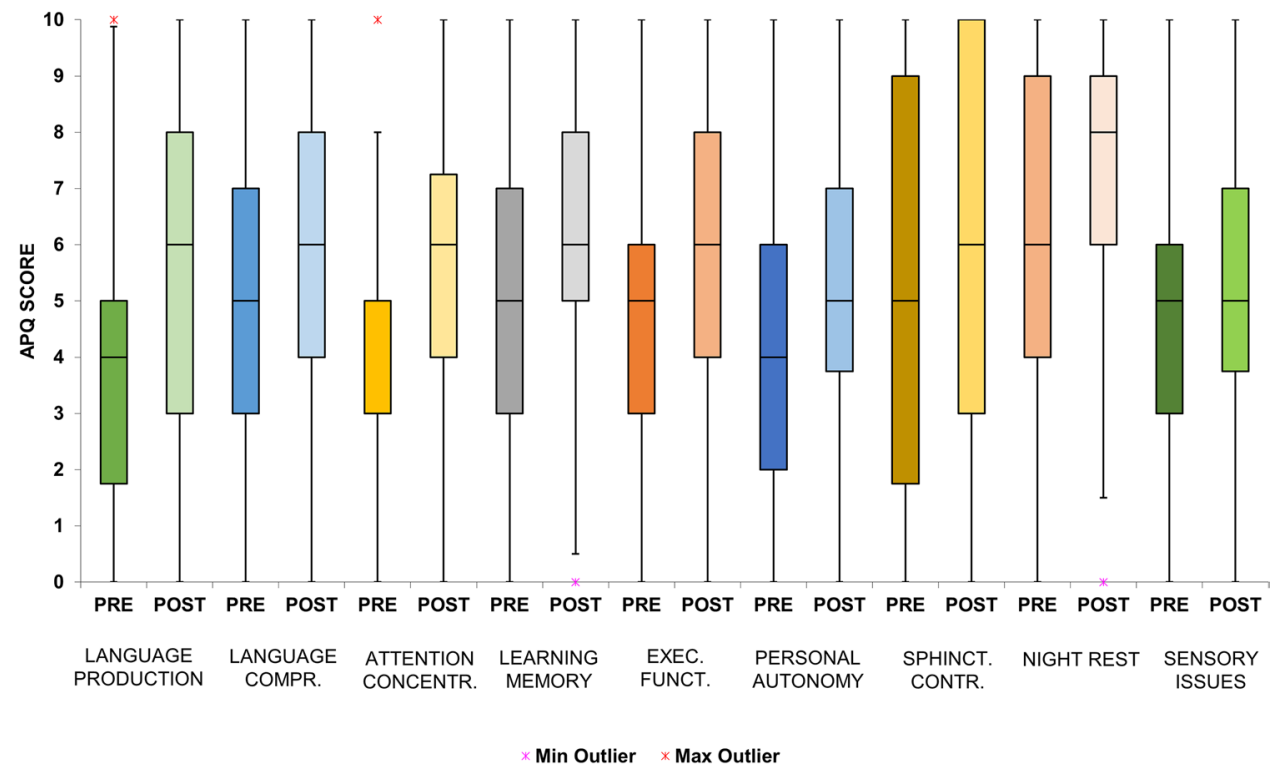

Sensory Issues

At T0, the Q1 value was 3, and the median value was 5; the Q3 value was 6, and the IQR was 3. At T6, the Q1 value was 3.75, and the median value was 5; the Q3 value was 7, and the IQR was 3.25 (Fig. 2). Statistical significance was set at $P$-values of $<0.05$.

\section{Cluster Behavioral Skills and Symptoms}

\section{Non-verbal Communication Skills}

At T0, the Q1 value was 4, and the median value was 5; the Q3 value was 7, and the IQR was 3. At T6, the Q1 value was 5, and the median value was 7; the Q3 value was 8, and the IQR was 3 (Fig. 3). Statistical significance was set at $P$-values of $<0.05$.

\section{Voluntary Eye Movements and Eye Contact}

At T0, the Q1 value was 3.75, and the median value was 5; the Q3 value was 7, and the IQR was 3.25. At T6, the $\mathrm{Q} 1$ value was 4, and the median value was 6; the Q3 value was 8 , and the IQR was 4 (Fig. 3). Statistical significance was set at $P$-values of $<0.05$.

\section{Adaptation to Changes}

At T0, the Q1 value was 4, and the median value was 5; the Q3 value was 7, and the IQR was 3. At T6, the Q1 value was 5 , and the median value was 6 ; the $\mathrm{Q} 3$ value the IQR was 3 (Fig. 2). Statistical significance was set at $P$-values of $<0.05$. 
Fig. 3 Behavioral skills show an improvement with statistical significance $p<0.5$

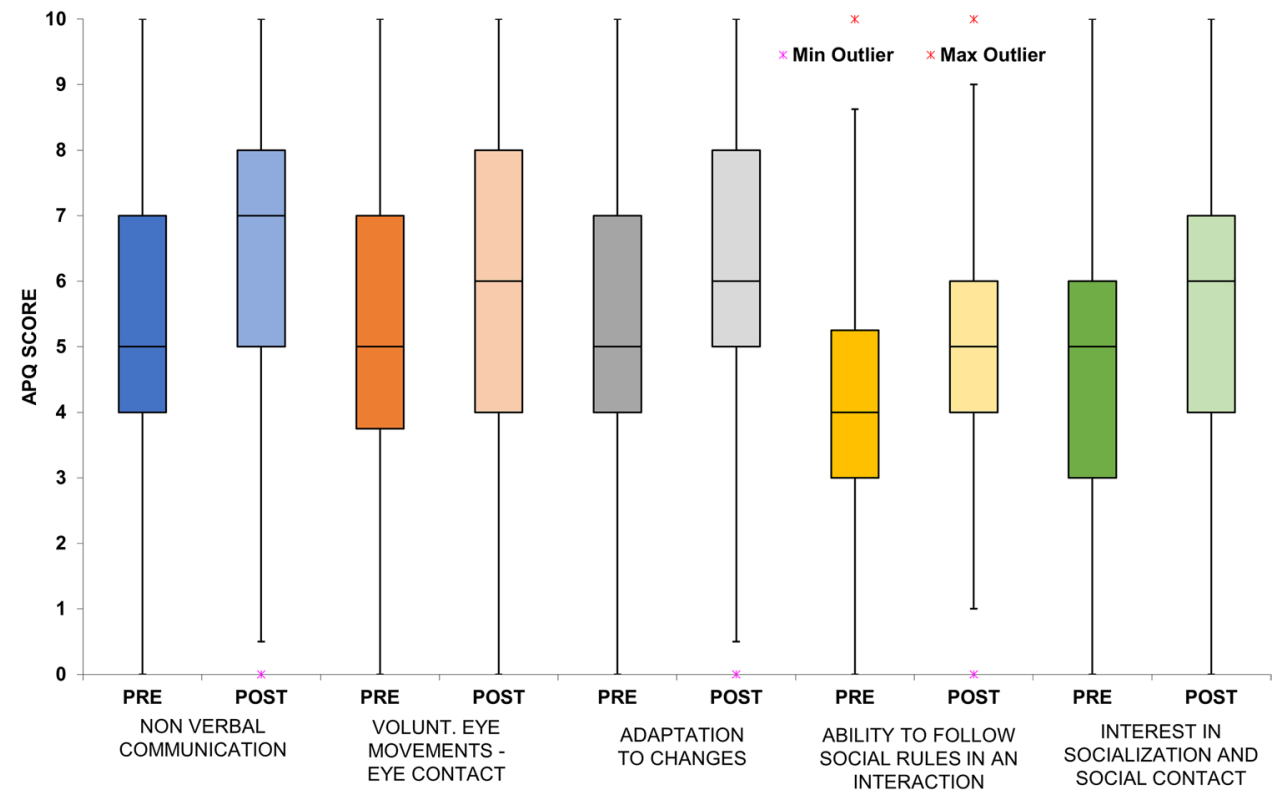

was 8 , and the IQR was 3 (Fig. 3). Statistical significance was set at $P$-values of $<0.05$.

\section{Ability to Follow Social Rules in an Interaction}

At T0, the Q1 value was 3, and the median value was 4; the Q3 value was 5.25, and the IQR was 2.25. At T6, the $\mathrm{Q} 1$ value was 4, and the median value was 5; the Q3 value was 6, and the IQR was 2 (Fig. 3). Statistical significance was set at $P$-values of $<0.05$.

\section{Interest in Socialization and Social Contact}

At T0, the Q1 value was 3, and the median value was 5; the $\mathrm{Q} 3$ value was 6, and the IQR was 3. At T6, the Q1 value was 4, and the median value was 6; the Q3 value was 7, and the IQR was 3 (Fig. 3). Statistical significance was set at $P$-values of $<0.05$.

\section{Self-aggressive Behaviors}

At T0, the Q1 value was 0 , and the median value was 2; the Q3 value was 4, and the IQR was 4. At T6, the Q1 value was 0 , and the median value was 1 ; the Q3 value was 3, and
Fig. 4 Behavioral symptoms show an improvement with statistical significance $p<0.5$

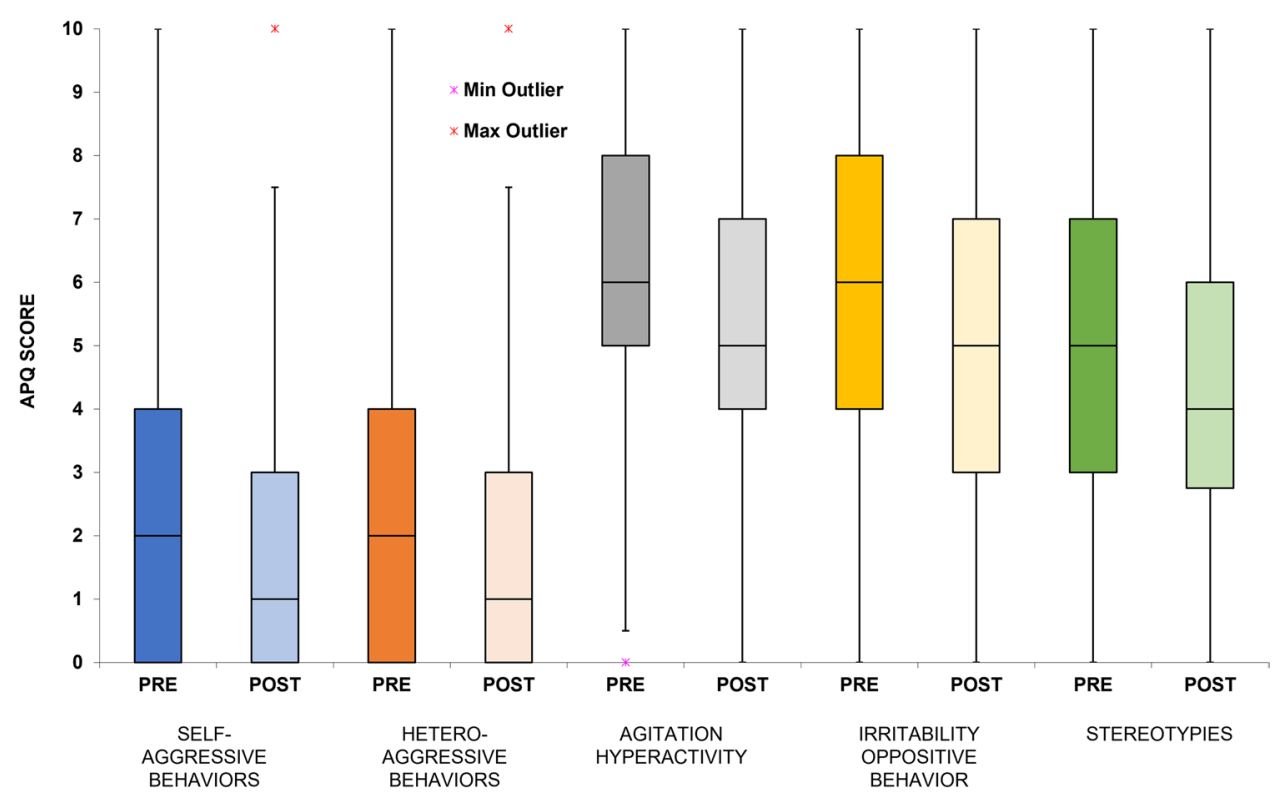


the IQR was 3 (Fig. 4). Statistical significance was set at $P$-values of $<0.5$.

\section{Hetero-aggressive Behaviors}

At T0, the Q1 value was 0, and the median value was 2; the Q3 value was 4, and the IQR was 4. At T6, the Q1 value was 0 , and the median value was 1 ; the $\mathrm{Q} 3$ value was 3 , and the IQR was 3 (Fig. 4). Statistical significance was set at $P$-values of $<0.5$.

\section{Agitation and Hyperactivity}

At T0, the Q1 value was 5, and the median value was 6; the Q3 value was 8, and the IQR was 3. At T6, the Q1 value was 4, and the median value was 5; the Q3 value was 7, and the IQR was 3 (Fig. 4). Statistical significance was set at $P$-values of $<0.5$.

\section{Irritability and Oppositive Behaviors}

At T0, the Q1 value was 4, and the median value was 6; the $\mathrm{Q} 3$ value was 8, and the IQR was 4. At T6, the Q1 value was 3, and the median value was 5; the Q3 value was 7, and the IQR was 4 (Fig. 4). Statistical significance was set at $P$-values of $<0.5$.

\section{Stereotyped Behaviors}

At T0, the Q1 value was 3, and the median value was 5; the Q3 value was 7, and the IQR was 4. At T6, the Q1 value was 2.75 , and the median value was 4; the $\mathrm{Q} 3$ value was 6 , and the IQR was 3.25 (Fig. 4). Statistical significance was set at $P$-values of $<0.5$.

\section{Adverse Events}

No adverse events were reported by the participants or observed by the investigators.

\section{Discussion}

Internalizing problems are characterized by anxiety, depressive symptoms, social withdrawal, and somatic complaints (Gobel et al., 2016). The scientific literature provides ample support to the fact that the comorbidity of internalizing disorders, such as depression (DeFilippis, 2018; Gegelashvili, 2019), anxiety (Kim et al., 2021; Vasa et al., 2020), and stress-related disorders (Corbett et al., 2021; Taylor et al., 2021), in ASD can affect the deficit severity, symptomatologic expression, and overall quality of life of individuals with ASD.
On these premises, the use of NIBS methods for treating ASD is growing, and studies conducted with these techniques have reported positive effects on social, health, and behavioral problems in individuals with ASD (Khaleghi et al., 2020). Existing evidence demonstrates that NIBS methods could be helpful in treating some dimensions of ASD, such as repetitive and stereotyped behaviors, sociability, or some aspects of executive and cognitive functions (Khaleghi et al., 2020). The global effects induced by REAC NPO and NPPO/NPPO-CB treatments in individuals with ASD can be contextualized in studies reporting the positive effects of NIBS methods on ASD-related behavioral, cognitive, and neurophysiological dysfunctions. In fact, the results obtained in this research demonstrate a positive correlation between the REAC NPO and NPPO treatments and improvement in the typical deficits of ASD perceived by caregivers at approximately 3 months after the end of the treatment cycle. Even in individuals with ASD, a single REAC treatment cycle has been able to mitigate the impact of internalizing disorders, as already proven in a non-ASD population (Rinaldi et al., 2019). This effect allowed the reduction of the severity of some ASD deficits and symptoms, optimizing the existing skills and improving the mental health conditions and functional outcomes of patients.

A comparison of the results obtained using different NIBS methods is very difficult because of the very large heterogeneity and variability among studies in terms of patients' profiles, study designs, schedules, and stimulation parameters (Khaleghi et al., 2020).

Moreover, while demonstrating its effectiveness in the same ASD dimensions on which other NIBS techniques are effective, the REAC technology differs in its mechanism of action. In fact, other NIBS methods yield their effects through administration on specific brain areas, depending on their hypotheses regarding the origin of the neural ASD impairments; their capacity to evoke neuroplasticity effects is related to the stimulation site, intensity, and frequency (Khaleghi et al., 2020) because their actions are carried out by inducing inhibitory or excitatory stimulations.

The REAC technology has not a main target brain region for stimulation because it is able to globally reorganize the level of synaptic activities and the related neural circuitry dynamics in the brain (Zippo et al., 2015), not eliciting specific inhibitory or excitatory stimulations, but optimizing EBA and rearranging neural dynamics within "opportune" ranges when altered.

\section{Limitation and Future Research}

One of the major limitations of this study is the lack of a control group and the fact that one of the two verification tests involves a parental self-report method. Further studies are needed to better understand the effects of multiple 
treatment cycles with appropriate follow-up periods. This study suggests promoting further research to verify the effects of REAC NPO and NPPO/NPPO-CB treatments in low-functioning individuals toward high functionality and on relational linguistic skills.

Acknowledgements We thank Dr. Alessandra Cappelli and Dr. Ing. Matteo Lotti Margotti for their precious cooperation.

Author Contribution AR designed and executed the study, assisted with the data analyses, and wrote the manuscript. MCMM, PCFC, NAPOS, JAVM, VF, and SR designed the study and wrote the manuscript. MM analyzed the data and wrote a part of the results. AR, MM, $\mathrm{VF}$, and SR wrote and edited the final manuscript.

Data Availability All raw data are available on request in the form of an Excel file.

\section{Declarations}

Ethics Statement This research was approved by the Institutional Review Board of the Centre for Developmental Biology and Reprogramming of the University of Sassari, Italy, and performed in accordance with the ethical standards as laid down in the 1964 Declaration of Helsinki and its later amendments or comparable ethical standards.

Informed Consent Informed consent was obtained from all parents after a detailed explanation of the treatments, granting researchers' permission to access patient data for the purpose of scientific study and publication while ensuring patient anonymity.

Conflict of Interest Financial interests: SR and VF are authors of the REAC technology patent. The other authors declare no conflicts of interest regarding the publication of this paper.

Non-financial interests: AR is the daughter of SR and VF.

Open Access This article is licensed under a Creative Commons Attribution 4.0 International License, which permits use, sharing, adaptation, distribution and reproduction in any medium or format, as long as you give appropriate credit to the original author(s) and the source, provide a link to the Creative Commons licence, and indicate if changes were made. The images or other third party material in this article are included in the article's Creative Commons licence, unless indicated otherwise in a credit line to the material. If material is not included in the article's Creative Commons licence and your intended use is not permitted by statutory regulation or exceeds the permitted use, you will need to obtain permission directly from the copyright holder. To view a copy of this licence, visit http://creativecommons.org/licenses/by/4.0/.

\section{References}

Adams, D., \& Emerson, L. M. (2021). The impact of anxiety in children on the autism spectrum. Journal of Autism and Developmental Disorders, 51(6), 1909-1920. https://doi.org/10.1007/ s10803-020-04673-3

Amatachaya, A., Auvichayapat, N., Patjanasoontorn, N., Suphakunpinyo, C., Ngernyam, N., Aree-Uea, B., ... Auvichayapat, P. (2014). Effect of anodal transcranial direct current stimulation on autism: A randomized double-blind crossover trial. Behavioral Neurology, 2014, 173073. https://doi.org/10.1155/2014/173073

Baer, M. L., \& Colello, R. J. (2016). Endogenous bioelectric fields: A putative regulator of wound repair and regeneration in the central nervous system. Neural Regeneration Research, 11(6), 861-864. https://doi.org/10.4103/1673-5374.184446

Becker, E. B., \& Stoodley, C. J. (2013). Autism spectrum disorder and the cerebellum. International Review of Neurobiology, 113, 1-34. https://doi.org/10.1016/B978-0-12-418700-9.00001-0

Chernet, B., \& Levin, M. (2013). Endogenous voltage potentials and the microenvironment: Bioelectric signals that reveal, induce and normalize cancer. Journal of Clinical \& Experimental Oncology, (Supplement 1). https://doi.org/10.4172/2324-9110.S1-002

Corbett, B. A., Muscatello, R. A., Klemencic, M. E., \& Schwartzman, J. M. (2021). The impact of COVID-19 on stress, anxiety, and coping in youth with and without autism and their parents. Autism Research. https://doi.org/10.1002/aur.2521

D’Mello, A. M., Crocetti, D., Mostofsky, S. H., \& Stoodley, C. J. (2015). Cerebellar gray matter and lobular volumes correlate with core autism symptoms. Neuroimage Clinical, 7, 631-639. https://doi.org/10.1016/j.nicl.2015.02.007

D’Mello, A. M., \& Stoodley, C. J. (2015). Cerebro-cerebellar circuits in autism spectrum disorder. Frontiers in Neuroscience, 9, 408. https://doi.org/10.3389/fnins.2015.00408

DeFilippis, M. (2018). Depression in children and adolescents with autism spectrum disorder. Children (Basel, Switzerland), 5(9). https://doi.org/10.3390/children5090112

Enticott, P. G., Kirkovski, M., \& Oberman, L. M. (2019). Transcranial magnetic stimulation in autism spectrum disorder. In L. M. Oberman \& P. G. Enticott (Eds.), Neurotechnology and brain stimulation in pediatric psychiatric and neurodevelopmental disorders (pp. 83-113). Academic Press. https://doi.org/10. 1016/b978-0-12-812777-3.00005-2

Fontani, V., Mannu, P., Castagna, A., \& Rinaldi, S. (2011a). Social anxiety disorder: Radio electric asymmetric conveyor brain stimulation versus sertraline. Patient Preference and Adherence, 5, 581-586. https://doi.org/10.2147/PPA.S27409

Fontani, V., Rinaldi, S., Aravagli, L., Mannu, P., Castagna, A., \& Margotti, M. L. (2011b). Noninvasive radioelectric asymmetric brain stimulation in the treatment of stress-related pain and physical problems: Psychometric evaluation in a randomized, single-blind placebo-controlled, naturalistic study. International Journal of General Medicine, 4, 681-686. https://doi.org/10. 2147/IJGM.S24628

Fontani, V., Rinaldi, S., Castagna, A., \& Margotti, M. L. (2012). Noninvasive radioelectric asymmetric conveyor brain stimulation treatment improves balance in individuals over 65 suffering from neurological diseases: Pilot study. Therapeutics and Clinical Risk Management, 8, 73-78. https://doi.org/10.2147/ TCRM.S28812

Garcia-Gonzalez, S., Lugo-Marin, J., Setien-Ramos, I., GisbertGustemps, L., Arteaga-Henriquez, G., Diez-Villoria, E., \& Ramos-Quiroga, J. A. (2021). Transcranial direct current stimulation in Autism Spectrum Disorder: A systematic review and meta-analysis. European Neuropsychopharmacoly. https://doi.org/ 10.1016/j.euroneuro.2021.02.017

Gegelashvili, M. (2019). Autism and depression (review). Georgian Medical News(292-293), 54-56. https://www.ncbi.nlm.nih.gov/ pubmed/31560663

Gobel, A., Henning, A., Moller, C., \& Aschersleben, G. (2016). The relationship between emotion comprehension and internalizing and externalizing behavior in 7- to 10-year-old children. Frontiers in Psychology, 7, 1917. https://doi.org/10.3389/fpsyg.2016.01917

Hampson, D. R., \& Blatt, G. J. (2015). Autism spectrum disorders and neuropathology of the cerebellum. Frontiers in Neuroscience, 9(420), 420. https://doi.org/10.3389/fnins.2015.00420 
Hashimoto, T., Tayama, M., Murakawa, K., Yoshimoto, T., Miyazaki, M., Harada, M., \& Kuroda, Y. (1995). Development of the brainstem and cerebellum in autistic patients. Journal of Autism and Developmental Disorders, 25(1), 1-18. https://doi.org/10.1007/ BF02178163

Johnson, B., Stanley-Cary, C., Fielding, J., Rinehart, N., \& Papadopoulos, N. (2014). Cerebellum and the psychopathology of autism and Asperger's disorder. In V. B. Patel, V. R. Preedy, \& C. R. Martin (Eds.), Comprehensive guide to autism (pp. 845-863). Springer, New York. https://doi.org/10.1007/978-1-4614-4788-7_44

Khaleghi, A., Zarafshan, H., Vand, S. R., \& Mohammadi, M. R. (2020). Effects of non-invasive neurostimulation on autism spectrum disorder: A systematic review. Clinical Psychopharmacology and Neuroscience, 18(4), 527-552. https://doi.org/10.9758/cpn.2020. 18.4.527

Kim, S. Y., Kim, Y. A., Song, D. Y., Bong, G., Kim, J. M., Kim, J. H., \& Yoo, H. J. (2021). State and trait anxiety of adolescents with autism spectrum disorders. Psychiatry Investigation, 18(3), 257-265. https://doi.org/10.30773/pi.2020.0328

Levin, M. (2014). Molecular bioelectricity: How endogenous voltage potentials control cell behavior and instruct pattern regulation in vivo. Molecular Biology of the Cell, 25(24), 3835-3850. https:// doi.org/10.1091/mbc.E13-12-0708

Levin, M., Pezzulo, G., \& Finkelstein, J. M. (2017). Endogenous bioelectric signaling networks: Exploiting voltage gradients for control of growth and form. Annual Review of Biomedical Engineering, 19, 353-387. https://doi.org/10.1146/annurev-bioeng-071114-040647

Lorenzini, L., Giuliani, A., Sivilia, S., Baldassarro, V. A., Fernandez, M., Lotti Margotti, M., ... Calza, L. (2016). REAC technology modifies pathological neuroinflammation and motor behaviour in an Alzheimer's disease mouse model. Scientific Reports, 6(1), 35719. https:// doi.org/10.1038/srep35719

Maioli, M., Rinaldi, S., Santaniello, S., Castagna, A., Pigliaru, G., Delitala, A., ... Ventura, C. (2014). Anti-senescence efficacy of radioelectric asymmetric conveyer technology. Age (Dordr), 36(1), 9-20. https://doi.org/10.1007/s11357-013-9537-8

Maioli, M., Rinaldi, S., Santaniello, S., Castagna, A., Pigliaru, G., Gualini, S., ... Ventura, C. (2013). Radio electric conveyed fields directly reprogram human dermal skin fibroblasts toward cardiac, neuronal, and skeletal muscle-like lineages [Research Support, Non-U.S. Gov't]. Cell Transplantation, 22(7), 1227-1235. https://doi.org/10. 3727/096368912X657297

Maioli, M., Rinaldi, S., Santaniello, S., Castagna, A., Pigliaru, G., Gualini, S., ... Ventura, C. (2012). Radiofrequency energy loop primes cardiac, neuronal, and skeletal muscle differentiation in mouse embryonic stem cells: A new tool for improving tissue regeneration. Cell Transplantation, 21(6), 1225-1233. https://doi.org/10. 3727/096368911X600966

Manto, M. (2009). Mechanisms of human cerebellar dysmetria: Experimental evidence and current conceptual bases. Journal of NeuroEngineering and Rehabilitation, 6, 10. https://doi.org/10.1186/ 1743-0003-6-10

Mura, M., Castagna, A., Fontani, V., \& Rinaldi, S. (2012). Preliminary pilot fMRI study of neuropostural optimization with a noninvasive asymmetric radioelectric brain stimulation protocol in functional dysmetria. Neuropsychiatric Disease and Treatment, 8, 149-154. https://doi.org/10.2147/NDT.S29971

Oerbeck, B., Overgaard, K. R., Attwood, T., \& Bjaastad, J. F. (2021). "Less stress": A pilot study on a cognitive behavioral treatment program for anxiety in children with autism spectrum disorders. Scandinavian Journal of Child and Adolescent Psychiatry and Psychology, 9, 30-40. https://doi.org/10.21307/sjcapp-2021-005

Olazaran, J., Gonzalez, B., Lopez-Alvarez, J., Castagna, A., Osa-Ruiz, E., Herrero-Cano, V., ... Martinez-Martin, P. (2013). Motor effects of REAC in advanced Alzheimer's disease: Results from a pilot trial.
Journal of Alzheimer's Disease, 36(2), 297-302. https://doi.org/10. 3233/JAD-130077

Olazaran, J., Gonzalez, B., Osa-Ruiz, E., Felipe-Ruiz, S., Boyano, I., Fontani, V., ... Martinez-Martin, P. (2014). Motor effects of radio electric asymmetric conveyer in Alzheimer's disease: Results from a cross-over trial. Journal of Alzheimer's Disease, 42(1), 325-332. https://doi.org/10.3233/JAD-140417

Olivieri, E. B., Vecchiato, C., Ignaccolo, N., Mannu, P., Castagna, A., Aravagli, L., ... Rinaldi, S. (2011). Radioelectric brain stimulation in the treatment of generalized anxiety disorder with comorbid major depression in a psychiatric hospital: A pilot study. Neuropsychiatric Disease and Treatment, 7, 449-455. https://doi.org/10.2147/NDT. S23420

Panaro, M. A., Aloisi, A., Nicolardi, G., Lofrumento, D. D., De Nuccio, F., La Pesa, V., ... Rinaldi, S. (2018). Radio electric asymmetric conveyer technology modulates neuroinflammation in a mouse model of neurodegeneration. Neuroscience Bulletin, 34(2), 270-282. https:// doi.org/10.1007/s12264-017-0188-0

Parsons, P. A. (1990). Fluctuating asymmetry: An epigenetic measure of stress. Biological Review of the Cambridge Philosophical Society, 65(2), 131-145. https://doi.org/10.1111/j.1469-185x.1990.tb011 86.x

Pinheiro Barcessat, A. R., Nolli Bittencourt, M., Duarte Ferreira, L., de Souza Neri, E., Coelho Pereira, J. A., Bechelli, F., \& Rinaldi, A. (2020a). REAC cervicobrachial neuromodulation treatment of depression, anxiety, and stress during the COVID-19 pandemic. Psychology Research and Behavior Management, 13(13), 929-937. https://doi.org/10.2147/PRBM.S275730

Pinheiro Barcessat, A. R., NolliBittencourt, M., Goes Goncalves, R., de Oliveira, Goncalves, Cruz, A. V., Coelho Pereira, J. A., Bechelli, F. A., \& Rinaldi, A. (2020). REAC neuromodulation treatments in depression, anxiety and stress. A comparative retrospective study. Psychology Research and Behavior Management, 13(13), 12471256. https://doi.org/10.2147/PRBM.S287143

Rinaldi, A., Rinaldi, C., Coelho Pereira, J. A., Lotti Margotti, M., Bittencourt, M. N., Barcessat, A. R. P., ... Rinaldi, S. (2019). Radio electric asymmetric conveyer neuromodulation in depression, anxiety, and stress. Neuropsychiatric Disease and Treatment, 15, 469-480. https://doi.org/10.2147/NDT.S195466

Rinaldi, S., Calza, L., Giardino, L., Biella, G. E., Zippo, A. G., \& Fontani, V. (2015). Radio electric asymmetric conveyer: A novel neuromodulation technology in Alzheimer's and other neurodegenerative diseases. Frontiers in Psychiatry, 6, 22. https://doi.org/10.3389/fpsyt. 2015.00022

Rinaldi, S., Fontani, V., Aravagli, L., \& Margotti, M. L. (2010). Psychological and symptomatic stress-related disorders with radio-electric treatment: Psychometric evaluation. Stress and Health, 26(5), 350358. https://doi.org/10.1002/smi.1298

Rinaldi, S., Maioli, M., Pigliaru, G., Castagna, A., Santaniello, S., Basoli, V., ... Ventura, C. (2014). Stem cell senescence. Effects of REAC technology on telomerase-independent and telomerase-dependent pathways. Scientific Reports, 4, 6373. https://doi.org/10.1038/srep0 6373

Rinaldi, S., Mura, M., Castagna, A., \& Fontani, V. (2014). Long-lasting changes in brain activation induced by a single REAC technology pulse in Wi-Fi bands. Randomized double-blind fMRI qualitative study. Scientific Reports, 4, 5668. https://doi.org/10.1038/srep05668

Taylor, E. C., Livingston, L. A., Callan, M. J., Ashwin, C., \& Shah, P. (2021). Autonomic dysfunction in autism: The roles of anxiety, depression, and stress. Autism, 25(3), 744-752. https://doi.org/10. $1177 / 1362361320985658$

Timmann, D., \& Daum, I. (2007). Cerebellar contributions to cognitive functions: A progress report after two decades of research. Cerebellum, 6(3), 159-162. https://doi.org/10.1080/14734220701496448

Tuszynski, J. A. (2019). The bioelectric circuitry of the cell. In S. Makarov, M. Horner, \& G. Noetscher (Eds.), Brain and human 
body modeling: Computational human modeling at EMBC 2018 (pp. 195-208). Springer International Publishing. https://doi.org/10. 1007/978-3-030-21293-3_11

Vasa, R. A., Keefer, A., McDonald, R. G., Hunsche, M. C., \& Kerns, C. M. (2020). A scoping review of anxiety in young children with autism spectrum disorder. Autism Research, 13(12), 2038-2057. https://doi.org/10.1002/aur.2395

Zippo, A. G., Rinaldi, S., Pellegata, G., Caramenti, G. C., Valente, M., Fontani, V., \& Biella, G. E. (2015). Electrophysiological effects of non-invasive Radio Electric Asymmetric Conveyor (REAC) on thalamocortical neural activities and perturbed experimental conditions. Scientic Reports, 5, 18200. https://doi.org/10.1038/srep18200

Publisher's Note Springer Nature remains neutral with regard to jurisdictional claims in published maps and institutional affiliations. 